\title{
Spontaneous adaptation of ion selectivity in a bacterial flagellar motor
}

2 Pietro Ridone ${ }^{1}$, Tsubasa Ishida ${ }^{2}$, Angela Lin ${ }^{1}$, David T Humphreys ${ }^{3}$, Eleni

3 Giannoulatou $^{3}$, Yoshiyuki Sowa ${ }^{2,4}$, Matthew A. B. Baker ${ }^{1,5^{*}}$

${ }^{1}$ School of Biotechnology and Biomolecular Sciences, University of New South Wales, Sydney, Australia.

${ }^{2}$ Department of Frontier Bioscience, Hosei University, Tokyo, Japan.

${ }^{3}$ Victor Chang Cardiac Research Institute, Sydney, Australia

${ }^{4}$ Research Center for Micro-Nano Technology, Hosei University, Tokyo, Japan.

${ }^{5}$ CSIRO Synthetic Biology Future Science Platform, Brisbane, Australia.

*correspondence: matthew.baker@unsw.edu.au

\section{ABSTRACT}

Motility provides a selective advantage to many bacterial species and is often achieved by rotation of flagella that propel the cell towards more favourable conditions. In most species, the rotation of the flagellum, driven by the Bacterial Flagellar Motor (BFM), is powered by $\mathrm{H}^{+}$or $\mathrm{Na}^{+}$ion transit through the torque-generating stator subunit of the motor complex. The ionic requirements for motility appear to have adapted to environmental changes throughout history but the molecular basis of this adaptation remains unknown. Here we used CRISPR engineering to replace the native Escherichia coli $\mathrm{H}^{+}$-powered stator with $\mathrm{Na}^{+}$-powered stator genes and report the rapid and spontaneous reversion to $\mathrm{H}^{+}$-power in a low sodium environment. We follow the evolution of the stators during their reversion to $\mathrm{H}^{+}$-powered motility and use whole genome sequencing to identify both flagellar- and non-flagellar-associated genes involved in the cell's adaptation to new power sources. This work highlights the utility of the flagellar stator system for studying the molecular mechanisms underlying adaptation and demonstrates how environmental change can rapidly alter the function of an ion transporter. 


\section{INTRODUCTION}

Bacterial motility via the flagellar motor represents one of the earliest forms of locomotion (Miyata et al., 2020). This rotary motility imparts such significant selective advantage (Roszak and Colwell 1987, Fraebel, Mickalide et al. 2017) that resources are allocated to chemotaxis even in the absence of nutrient gradients (Gude, Pince et al. 2020, Ni, Colin et al. 2020). Furthermore, the evolutionary origins, and subsequent adaptation of the motor are of significant scientific and public interest (Pallen and Matzke 2006), since the BFM holds prominence as an ancient, large, molecular complex of high sophistication.

The torque that drives the BFM is supplied by motor-associated transmembrane protein-complexes known as stators. The stator complex, an asymmetric heteroheptamer (in E. coli: $\operatorname{Mot} \mathrm{A}_{5} \mathrm{Mot}_{2}$ ) most likely acts itself as a miniature rotating nanomachine coupling ion transit to rotation (Deme, Johnson et al. 2020, Santiveri, Roa-Eguiara et al. 2020). The stators are essential for motility, as they drive rotation, and are accessible for studies in experimental evolution due to their unambiguous role in connecting a specific environmental cue (presence of the coupling ion) to an easily discernible phenotype (cell swimming). Furthermore, the stators have been subject to protein engineering approaches for many years, in particular the synthesis of chimeric stator constructs that enable the motor of $E$. coli, natively proton-driven, to be powered by sodium ion flow (Asai, Yakushi et al. 2003). The majority of stators are proton driven, but many that are sodium-driven can be found in nature, and this divergence is presumed to have occurred in the distant past (Takekawa, Isumi et al. 2019, Islam, Lin et al. 2020, Lai, Ridone et al. 2020). Past reports have argued that $\mathrm{H}^{+}$-coupled motility diverged from $\mathrm{Na}^{+}$-coupled machinery in ancestral times (Takekawa, Nishiyama et al. 2015), but the molecular basis for this adaptation, and the evolutionary landscape that constrains stator adaptation remains unclear.

In order to simulate the effects of natural evolution on stator adaptation we designed an experiment where an E. coli strain, expressing only a sodium-powered stator, would be introduced to a non-lethal environment (soft agar swim plate) which lacked the power source for the stator $\left(\mathrm{Na}^{+}\right)$. Our hypothesis was that the population would undergo selection for upmotile variants, adapting its stators to function in the new environment. 
We used genomic editing techniques (no-SCAR CRISPR/Cas9 (Reisch and Prather 2015) and $\lambda$-Red (Datsenko and Wanner 2000)) to replace the native mot $A$ motB stator genes of the E. coli BFM with chimeric sodium-powered pomA potB (henceforth pots) stator genes derived from Vibrio alginolyticus (Asai, Yakushi et al. 2003). We transplanted the pots stator genes at the same location and orientation of the native motA motB locus to preserve the native genomic context of the motile RP437 E. coli strain. We then examined which genetic changes occurred during growth on soft-agar in depleted sodium, that is, under selective pressure for proton-driven motility. We performed our directed evolution experiments of our pots $E$. coli strain in the absence of antibiotics to avoid additional, undesired selective pressures (Baym, Lieberman et al. 2016).

\section{RESULTS}

The RP437 strain was edited to carry the pots stator genes in place of the native $E$. coli motAmotB genes (Fig. 1A) via the no-SCAR Method (Reisch and Prather 2015) and traditional $\lambda$-Red recombineering respectively (Supplementary Fig. $1 \& 2$ ). A single no-SCAR Pots clone was selected and tested on swim plates (Supplementary Fig. 3) after verification of successful editing by colony PCR and Sanger Sequencing (Supplementary Fig. 4). The edited strain was able to swim on sodium-rich (85 mM $\mathrm{NaCl}$ ) soft agar plates but not on potassium-rich sodium-poor $(67 \mathrm{mM} \mathrm{KCl}, \sim 8 \mathrm{mM}$ $\left[\mathrm{Na}^{+}\right]$) plates (Supplementary Fig. 3A). This edited strain exhibited the same swimming behaviour as the control stator-less strain with motility restored via an inducible plasmid vector that could express the Pots construct (RP6894 $\Delta$ motAmotB + pSHU1234, hereby pPots).

We next challenged this pots strains to survive on $\mathrm{K}^{+}$based soft agar for prolonged periods (Supplementary Fig. 3B). Motile subpopulations arose spontaneously from inoculated colonies within a few days. Cells from the edge of these motile flares were passaged onto fresh swim agar for up to 5 passages at 3-4 days intervals (Supplementary Fig. 5). When multiple flares occurred in a single swim ring, each was individually passaged (Supplementary Fig. 3B), and could be recapitulated (Fig. 1). Directed evolution consistently generated swimming flares when pots clones were cultured on agar containing yeast extract and tryptone $\left(\sim 8 \mathrm{mM}\left[\mathrm{Na}^{+}\right]\right)$, but not on minimal media $\left(\sim 1 \mathrm{mM}\right.$ total $\left.\left[\mathrm{Na}^{+}\right]\right)$or when the pots construct was encoded on a plasmid (Supplementary Fig. 6). One pots strain generated using $\lambda$-Red methods 
(Datsenko and Wanner 2000), which carried the native V. alginolyticus pomA promoter, also successfully produced flares (Supplementary Fig. 7).

Lineages were selected for whole-genome sequencing (WGS) after a preliminary screening for mutations in the stator genes by Sanger-sequencing PCR amplicons spanning the genomic pomApotB locus (Fig. 1). Variant calling to the MG1655 reference genome was used to compare single nucleotide polymorphisms (SNPs) between members of the same lineage. Our intended pomApotB edit was the only difference between the RP437 and Pots genomes, indicating that neither no-SCAR nor $\lambda$-Red editing had resulted in off-target edits. 153 SNPs were called as variants between our experimental parent RP437 and the MG1655 reference which were shared across all lineage members (Supplementary Table 1).

Several lineages whose descendants could swim in reduced sodium had mutations at the pots locus (L3.3-4-5: PotB G20V; L6.4-5: PomA L183F; L8.3-4-5: PotB G20W). In contrast, lineages passaged only on $85 \mathrm{mM} \mathrm{Na}^{+}$agar ( $\mathrm{L} 1$ \& L2) accumulated mutations not in stators but in the flagellar components. Lineages passaged on $\sim 8 \mathrm{mM}$ sodiumpoor agar whose descendants could not swim (L4) had no mutations on any flagellar genes.

To determine which genes may be involved in the adaptation process, we performed RNAseq experiments to measure transcript levels for two lineages which evolved different stator mutations over different lengths of time (Fig. 2, Supplementary Table 2). These were: PomA L183F ( $\mathrm{Na}+$ powered phenotype, 14 days) and PotB G20V ( $\mathrm{H}+$ phenotype, 7 days). From these, we selected the common ancestor to the two lineages (Pots), the last lineage member before the mutation occurred (L5.3 and L3.2, the prefixation sample) and the first available member carrying that lineage mutation on the chromosome (L6.4 and L3.3, the fixed variant). Of note, L5.3 displayed improved swimming despite being isogenic to its parent strain, the Pots common ancestor. Member L3.2 of the G20V lineage, the immediate predecessor to L3.3, in contrast, was non-motile. We closely examined the transcripts mapped to the PomA/PotB locus in all samples and saw no significant enrichment of mutant transcripts above the background noise in the pre-fixation samples (Supplementary Fig. 8). This confirmed that a chromosomal mutation, rather than a RNA editing event, was responsible for the observed stator variants. To identify common processes which led to variant fixation in these two lineages we focused first on the differentially expressed genes present in both lineages (Fig. 2). We then performed pathway analysis to identify which 
biological processes were relevant to the shared genes and analysed which regulatory elements controlled their expression.

Both lineages increased expression of $I r h A$ at the pre-fixation point, which acts as an inhibitor of the flhDC flagellar master regulator. Expression response was dominated

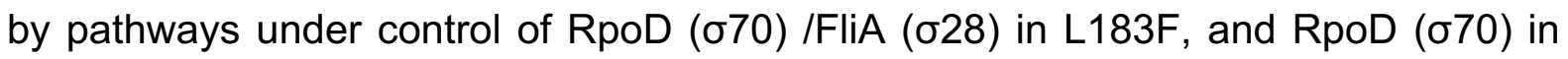
G20V (Supplementary Fig. 9). The prominence of FliA regulation in L183F was reflected by upregulation of motility genes at the fixation stage. Those same genes were not significantly affected in the G20V lineage, which instead promoted primarily adhesion/biofilm behavior in response to the changed environment.

We characterized rotational phenotypes of the parent and evolved strains in the presence and absence of sodium using a tethered cell assay with particular focus on the upmotile G20V variant, L3.3 (Fig. 3, Supplementary Fig. 10). The potB G20V fixed variant was clearly distinguishable from pots in this assay, and maintained rotation in both the absence of sodium and in the presence of the sodium blocker phenamil (Fig. $3 \mathrm{~A})$. We confirmed the PotB G20 $\mathrm{H}^{+}$-powered phenotype by introducing the same point mutation (GGG to GTG) on our plasmid vector (pPots) and testing motility in tethered cell assay when the protein was expressed in the stator-less $\triangle m o t A B$ RP6894 (Fig. 3B). Measurement of rotational speed vs lithium and sodium showed that the PotB-G20V variant was motile at $0 \mathrm{mM} \mathrm{Na}+$ and $0 \mathrm{mM} \mathrm{Li+}$, but showed a dependence on the concentration of ion presence, similar to pots, and in contrast to MotA/MotB (Supplementary Fig. 11A-C). All stator types had cessation of rotation at $50 \mu \mathrm{M} \mathrm{CCCP}$, and showed no measurable effect as $\mathrm{pH}$ was varied between 6.0 and 8.0 (Supplementary Fig. 11DE).

We further tracked single cell rotation in the tethered cell assay with sequential exchange of buffers (Supplementary Fig. 12). There, potB G20V (L3.3) actively rotated in sodium-free buffers and in presence of $100 \mu \mathrm{M}$ Phenamil - indicating sodium-free rotation and a disrupted phenamil binding site. We further synthesised all possible variants at site $\mathrm{G} 20$, and of these, only G20V was able to rotate in the absence of sodium (Supplementary Fig. 13).

We examined the natural prevalence of glycine and valine at site 20 across a phylogeny from a collection of 82 MotB sequences. Of these sequences, G20 was present only in the clade corresponding to Vibrio sp. PomB (7 sequences), whereas V20 was distributed across the phylogeny more broadly (41 sequences) 
(Supplementary Fig. 14). Ancestral reconstruction across every node of this phylogeny predicted that the ancestral phenotype was G20.

To examine mutation reproducibility in the stators at higher throughput, we subjected flares within the first 3 days of incubation, which were then passaged four more times at 3-day intervals. At the end of this experiment we selected the 20 terminal lineage members which produced the largest swim rings and Sanger-sequenced them at the pots locus. For these we observed a total of 5 mutations in pomA (S25C, D31N, P177A, P177Q and L183F) and one more new mutation in potB (L36Q) (Fig. 2B). We mapped monomer models for PomA and PotB generated using Alphafold (Mirdita, Schütze et al. 2021) to a model PomA ${ }_{5} P_{0} B_{2}$ complex using the recently published high-resolution B. subtilis Mot $\mathrm{A}_{5} \mathrm{~B}_{2}$ structure (Deme, Johnson et al. 2020) (Fig. 2C \& 2D). All stator mutations accumulated at sites proximal to or within the predicted iontransport pore, at the interface between the PotB transmembrane domain and the third and fourth transmembrane domains of PomA (Fig. 2D).

Over the course of these experiments, we found that three stator residues underwent mutation at the same site twice (PomA L183F (2x), PomA P177A \& P177Q, PotB G20V \& G20W). WGS revealed that the pitA gene had mutated in three separate lineages (L2.5, L3.5, L8.5) with one of the mutations occurring twice (PitA W112*Stop).

To test for the capacity for reversion, we took sequenced lineages that swam in $8 \mathrm{mM}$ $\left[\mathrm{Na}^{+}\right]$agar (L3.3-4-5, L6.4-5, L8.3-4-5) and reintroduced them to an environment with $85 \mathrm{mM}\left[\mathrm{Na}^{+}\right]$(Supplementary Fig. 15A). After 10 rounds of daily passaging, no reversion in the mutants that had enabled low-sodium swimming was observed, with only a single additional mutation gained: a potB T21A mutation in the terminal descendant of potB G20W pitA W112*stop (L8.5).

Finally, we tested whether evolution could be more easily directed on minimal media when starting from a more favourable vantage point. We examined all stator mutants that swam on $8 \mathrm{mM}\left[\mathrm{Na}^{+}\right]$plates in conditions of further sodium scarcity (minimal media: $1 \mathrm{mM}\left[\mathrm{Na}^{+}\right]$) (Supplementary Fig. 15B). Initially, only the strains with potB G20V and pomA P177Q mutations could swim, and this capacity was maintained following five passages over 12 days. Sanger sequencing revealed that the terminal descendant of potB G20V pitA G432R mutant (L3.5) gained a further mutation in pomA (M20L). A 
bioRxiv preprint doi: https://doi.org/10.1101/2021.01.26.427765; this version posted December $15,2021$. The copyright holder for this preprint (which was not certified by peer review) is the author/funder, who has granted bioRxiv a license to display the preprint in perpetuity. It is made available under aCC-BY 4.0 International license.

194 summary of all stator gene mutations obtained from all directed evolution experiments

195 is provided in Fig. 2B.

196 


\section{DISCUSSION}

Previous reports have shown that bacterial motility can adapt (Ni, Ghosh et al. 2017) and be rescued (Taylor, Mulley et al. 2015) via remodelling of the flagellar regulatory network. Ni et al. observed that evolutionary adaptation of motility occurs via remodeling of the checkpoint regulating flagellar gene expression (Ni, Ghosh et al. 2017). Their experiments tracked adaptive changes in swim plates, matching our experiments, however their only selection criteria were for improved swimming in an unhindered swimming population. In agreement with our results (fliM A161V), they found fliM (M67I and T192N) to be the amongst the first genes to mutate in the improved swimmer population but they did not report any changes in flgL ( $\triangle \mathrm{A} 57-\mathrm{Q} 58)$, nor, significantly, did they see any mutations in any stator genes. Flagellum-mediated motility also appears to be naturally robust to the loss of regulatory factors, such as the enhancer-binding protein fleQ in $P$. fluorescens, which function can be substituted by distantly related homologous proteins (Taylor, Mulley et al. 2015).

In contrast, our E. coli pots strain faced selective pressure from ion scarcity. Our scenario is reminiscent of previous semi-solid agar experimental evolution studies on the adaptation of antibiotic resistance and recapitulates similar results. In the MEGA plate experiments of Baym et al., they similarly saw that the phosphate transporter pitA was repeatedly mutated, often to a frameshifted or nonsense variant (Baym, Lieberman et al. 2016). In similar experiments the isocitrate dehydrogenase icd was also seen to mutate often (Fraebel, Mickalide et al. 2017, Anand, Olson et al. 2019).

Differential expression analysis of our RNAseq datasets revealed that our two chosen lineages underwent different transcriptomic pathways before fixing a mutation in the stator genes. While the L183F lineage displayed many upregulated genes involved in motility, the G20V lineage was found to regulate genes involved in biofilm formation.

The motile behaviour of the L5.3 member (pre-L183F mutation stage) was reflected by the upregulation of flagellar and chemotaxis genes. The genes involved have also been measured as upregulated in a similar study on the adaptation of $E$. coli to swimming in soft agar (Ni, Ghosh et al. 2017) which may explain how the strain can swim in very low $\mathrm{Na}+$ through upregulating several flagellar components including the chimeric sodium stators themselves (PomA and PotB). In contrast, the expression profile of the Na+-only swimmer L3.2 (pre-G20V mutation stage) was characterized by the regulation of genes involved in metabolic pathways indicative of nitrogen starvation, fermentation of products of catabolism such as amino acids and 
nucleotides and the transition to a biofilm lifestyle. Roughly $10 \%$ of genes showed significant changes in transcription in both lineage trios $(10.8 \%$ and $13.2 \%$ in $\mathrm{L} 183 \mathrm{~F}$ and $\mathrm{G} 20 \mathrm{~V}$ respectively) and all of these were upregulated during adaptation.

When the G20V mutation was fixed, L3.3 (G20V) was found to upregulate chemotaxis and motility genes, and other markers of adaptation to soft agar (cheA, $\operatorname{trp}, \operatorname{tar}, \operatorname{tap})(\mathrm{Ni}$, Ghosh et al. 2017). Both lineages upregulated the flhDC regulator LrhA at the premutation stage, suggesting that downregulation of the flagellar biosynthetic cascade is a shared trait contributing to stator adaptation. Similarly, both lineages upregulated nucleotide catabolic processes and salvage pathways, a feature also observed also in antibiotic response and which can affect mutation rates by disturbing the NTP balance in the cellular pool (Pang, McFaline et al. 2012, Foster, Lee et al. 2015, Lopatkin and Yang 2021).

Mutations in stators are known to affect ion usage and may confer dual-ion coupling capacity. For example, the substate preference of the B. alcalophilus MotPS stator $\left(\mathrm{Na}^{+} / \mathrm{K}^{+}\right.$and $\left.\mathrm{Rb}^{+}\right)$was changed with the single mutation M33L in MotS, causing the loss of both $\mathrm{K}^{+}$- and $\mathrm{Rb}^{+}$-coupling motility in E. coli (Terahara, Sano et al. 2012). Similarly a bi-functional B. clausii MotAB stator $\left(\mathrm{Na}^{+} / \mathrm{H}^{+}\right)$triple mutant (MotB V37L, A40S and G42S) was selective only for sodium ions while the combination of mutations G42S, Q43S and Q46A made MotB selective only for $\mathrm{H}^{+}$(Terahara, Krulwich et al. 2008). The previously reported S25C (Asai, Shoji et al. 2000) and D31N (Kojima, Shoji et al. 2000) amino acid substitutions in PomA have been shown to reduce motility and, in the case of $\mathrm{D} 31 \mathrm{~N}$, affect ion usage. Furthermore, single point mutations in stator genes of Vibrio spp. (eg. pomB G20V / G20R / P16S) have been shown to impart phenamil resistance both in Vibrio alginolyticus and in our pots strain (Jaques, Kim et al. 1999, Kojima, Asai et al. 1999, Ishida, Ito et al. 2019).

The PotB-G20V variant directly evolved here is capable of rotation in the total absence of sodium, however its swimming speed does increase with increased availability of either sodium or lithium, with an energisation profile more akin to the parental pots strain than the proton powered E. coli wild-type (Supplementary Fig. 11). This perhaps suggests that PotB-G20V is an opportunistic stator adaptation that enables promiscuity, allowing the passage of protons in sodium-scarce conditions.

The key difference in this work compared with previous efforts for directed evolution of the stators via mutagenesis (Garza, Harris-Haller et al. 1995, Kojima, Kuroda et al. 
$1999)$ is that here we edited the stators directly onto the $E$. coli genome to direct stator evolution in vivo in the native $E$. coli genomic context. This would not be not possible in Vibrio sp. since Vibrio cells do not survive at low sodium. Conversely, in our system it is difficult to use directed evolution to revert the ion-selectivity of the stator $(\mathrm{H}+$ to $\mathrm{Na}+$ ) because in $E$. coli it is not possible to drastically reduce the proton concentration without affecting confounding, essential systems. Nevertheless, the observation of no revertants agrees with previous work suggesting that requirements for $\mathrm{Na}^{+}$binding are more strict than for $\mathrm{H}^{+}$binding, and that mutations that convert a $\mathrm{Na}^{+}$motor to an $\mathrm{H}^{+}$ are more accessible than the reverse (Hase, Fedorova et al. 2001, Mulkidjanian, Galperin et al. 2008).

We observed a convergence of mutations on the stator genes and even to the very same nucleotide (GAG (L) to GAA $(F)$ in two separate pomA L183F lineages). Stator genes were the first to mutate in all of our WGS lineages under pressure for $\mathrm{H}^{+}$motility. Given this was from a clonal population under identical environmental constraints, it suggests that adaptation of the stators is prioritized in changing environments. Exactly how this may occur remains unclear. Mutation of pore-proximal residues into hydrophobic residues (eg. G20V) hinted at a mechanism for varying constrictions in the pore to alter the efficiency of ion binding. However, in contrast, none of the bulkier, hydrophobic amino acid replacements at PotB G20 (eg. F,W) resulted in a similar G20V-like $\mathrm{H}^{+}$-powered phenotype (Supplementary Fig. 13C). This suggests a selectivity mechanism enabled by G20V that is not driven simply by size. We propose an alternate mechanism whereby selectivity is maintained through perturbing the electrostatic environment in the vicinity of PotB D24 and the conserved P151 of PomA (E. coli MotA P173) (Mandadapu, Nirody et al. 2015).

Upon examination of the phylogenetic record with specific focus on the G20V locus, we observed that valine was more prevalent and distributed more broadly across microbial strains. This broader distribution, and ancestral sequence reconstruction across our phylogeny implied that the ancestral state of MotB was more to be likely V20. While G20V point mutations arose spontaneously in our experiments within a few days, these transitions do not appear to have occurred in the evolutionary record. This may indicate constraints on the adaptation of the sodium-powered stator units when considered in their native sodium-dependent hosts.

As noted by pioneers in directed evolution (Bloom and Arnold 2009), caution is 
selection pressures in the wild are not typically general. In this study we have leveraged our system, and our experimental design, to obtain large, measureable phenotypic change through a single mutation at the G20 locus. The stators of the flagellar motor appear ready to evolve in our experiments: they do not require transit through additional cryptic or neutral mutations, and thus are a model system for exploring molecular adaptation of ion selectivity. Alternative candidates such as sodium porters and pumps often have built in redundancy (Atia, McCloskey et al. 2016), and many marine microbes require sodium to be viable and thus evolutionary pressure cannot be applied with such specificity to a single protein complex. In our idealised system, we are able to examine isogenic bacterial colonies without competitive effects, in a homogeneous medium without local niches. Nevertheless, it still remains difficult to quantify rates of adaptation (McDonald 2019). For these reasons, our system is optimised to produce stator variants, but it may be that this ease for adaptation via single-point mutation is accessible precisely because cryptic mutations have been accumulated due to exposure to changing envinronments in the distant past (Draghi and Plotkin 2011, Hayden, Ferrada et al. 2011).

Overall, motility confers a fitness advantage that is worth significant energetic investment despite the high cost of synthesizing the flagellar machinery (Colin and Sourjik 2017, Ni, Colin et al. 2020). This advantage can only be seized if the correct ions are available for stator-conversion into torque, otherwise the cell appears to prioritize the adaptation of the stator genes to harness other available ions to restore function. CRISPR/Cas9 has become a widespread method used for precise genomic edits, yet the reversion of such edits appears rapid and gene targeted when focused selective pressure is applied. Here, ion scarcity supplied a strong selective pressure that allowed us to identify novel mutations which were correlated with altered ionspecificity. Our transplant of an unfit protein and the cells' rapid reversion of this edit demonstrates tight evolutionary regulation of the stator subunit in an ancient molecular complex.

\section{MATERIALS AND METHODS}

\section{E. coli strains, plasmids and culture media.}

E. coli strain RP437 was used as the parent strain for genomic editing experiments (Parkinson 1978, Block, Blair et al. 1989). The pSHU1234 (pPots) plasmid encoding pomA and potB (Ishida, Ito et al. 2019) was used as the template to generate the double stranded donor DNA. This was used to replace the $\operatorname{mot} A$ and $\operatorname{mot} B$ gene on 
the RP437 chromosome. Liquid cell culturing was done using LB broth $(\mathrm{NaCl}$ or $\mathrm{KCl}$, Yeast Extract, Bacto Tryptone). Cells were cultured on agar plates composed of LB Broth and 1\% Bacto Agar (BD, U.S.A.). Swim plate cultures were performed on the same substrates adjusted for agar content (0.3\% Bacto Agar). Minimal Media (MM) was used to replace Yeast Extract and Tryptone in soft agar swim plates. MM composition: $10 \mathrm{mM} \mathrm{KPO}_{4}, 1 \mathrm{mM}\left(\mathrm{NH}_{4}\right)_{2} \mathrm{SO}_{4}, 1 \mathrm{mM} \mathrm{MgSO}_{4}, 1 \mu \mathrm{g} / \mathrm{ml}$ Thiamine, $0.1 \mathrm{mM}$ of each of the amino acids Thr, Leu, His, Met and Ser.

\section{Editing E. coli with Cas9-assisted Recombineering.}

This procedure was adapted from the no-SCAR method (Reisch and Prather 2015) . The target strain to be edited (E. coli RP437) was sequentially transformed first with the pKD-sgRNA-3'MotA $\left(\mathrm{Sm}^{+}\right)$plasmid, encoding a sgRNA sequence directed the 3 ' end of $\operatorname{mot} A$, and then with the pCas9cr4 $\left(\mathrm{Cm}^{+}\right)$plasmid to yield a parent strain harboring both plasmids. The overlap extension PCR technique (Higuchi, Krummel et al. 1988) was employed to assemble linear double stranded DNA molecules (dsDNA) using 3 starting dsDNA fragments. The resulting donor DNA was electroporated in the plasmid-bearing host and the successfully edited clones were selected via colony PCR and Sanger sequencing of the motAB locus. A list of primers and PCR protocols used in this work is provided in Supplementary Fig. 16.

\section{Construction of Pots by $\lambda$-Red Recombineering}

Chromosomal replacement from motAmotB to pomApotB was achieved by using a $\lambda$ Red recombination system, with plasmid pKD46 encoding the Red system and positive selection for the recovery of swimming ability (Kinosita, Ishida et al. 2020). Motile clones were selected by isolating motile flares on swim plates.

\section{Tethered cell assay preparation and analysis}

The tethered cell assay with anti-FliC-antibody was performed as previously described (Nishiyama and Kojima 2012, Kinosita, Ishida et al. 2020). The tethered cells time lapse videos were recorded at 40x magnification on a phase contrast microscope (Nikon). Time lapse videos were collected using a camera (Chameleon3 CM3, Point Grey Research) recording 20s-long videos at 20 frames per second. Experiments involving single cell tracking during buffer exchange were recorded at 60 frames per second. A custom LabView software (Ishida, Ito et al. 2019, Islam, Lin et al. 2020) was employed as previously reported to estimate specific rotational parameters of the tethered cells such as rotation frequency (speed), clockwise and counterclockwise 
bias and switching frequency. Visualization of the data was performed using Graph Pad Prism 8.

\section{Single Nucleotide Polymorphism (SNP) analysis}

Whole genome sequencing of 22 . coli strains was performed using a MiSeq $2 x$ $150 \mathrm{bp}$ chip on an Illumina sequencing platform. Sequencing was carried out at the Ramaciotti Centre for Genomics, Kensington and delivered as demultiplexed fastQ files (Quality Control: >80\% bases higher than Q30 at 2×150 bp). The SNP calling and analysis was performed using Snippy (Pertea, Pertea et al. 2015, Bush, Foster et al. 2020). The short reads from sequencing were aligned to the MG1655 reference $E$. coli genome (GenBank: U00096.2) and to a synthetic genome based on MG1655, edited to contain the Pots stator sequences from pPots (pomA/potB) at the motAB locus.

RNA was extracted from bacterial cultures inoculated with glycerol stocks of the relevant strains and grown in $\mathrm{K}+\mathrm{TB}$ broth at $30^{\circ} \mathrm{C}$ until $\mathrm{OD}_{600}=0.5$. Total RNA was extracted from a $0.5 \mathrm{ml}$ aliquot of the culture using the RNAeasy Protect Bacteria Mini Kit (74524, QIAGEN) with on-column DNAse digestion, as indicated in the manufacturer protocol. RNA quality was assessed using a TapeStation System (RNA ScreenTape, Agilent). All RNA samples selected for sequncing had an RNA Integrity Number $(\mathrm{RIN})>8$. Library preparation and sequencing were performed at the Ramaciotti Centre using the NextSeq 500 platform (Illumina) running for 150 cycle using a MID flowcell in paired-end read mode (2x75bp). Fastq files containing the RNAseq reads underwent quality control using FastQC (Andrews 2010) and then processed with FASTP (Version 0.20.1)(Chen, Zhou et al. 2018), to remove low quality reads and trim adaptor sequences. Reads were aligned to the Pots reference genome using HISAT2(Kim, Langmead et al. 2015), transcripts were assembled and quantified using STRINGTIE (Pertea, Pertea et al. 2015) and differential expression analysis was carried out using DESeq2 (Love, Huber et al. 2014). Heatmaps dendrograms were generated using the Heatmap2 function from the $R$ gplots package (Heatmap2). Complete clustering was performed using the Euclidean Distance method. All the analysis tools described above were run on the Galaxy webserver (https://usegalaxy.org/). Nucleotide variations that were present in motB and pomA were quantified from RNA-Seq data using the Rsamtools pileup function (Morgan M 2021). This involved the writing of custom $R$ scripts (available upon request) that compared Rsamtools output to the genome reference. 
We then performed pathway analysis to identify which biological processes were relevant to the shared genes using the EcoCyc database (Karp, Riley et al. 2002).

\section{Structural Modelling}

The Pom $A_{5} P_{0} B_{2}$ model was assembled by modelling each monomer using the Colabfold pipeline (Mirdita, Schütze et al. 2021), and by aligning the resulting monomers to each subunit of the $B$. subtilis $M_{0} A_{5} B_{2}$ structure (PDB:6YSL)(Deme, Johnson et al. 2020).

\section{Phylogenetics and Ancestral Reconstruction}

Phylogeny was generated with RAxML-HPC v.8 on XSEDE (Stamatakis 2014) through the CIPRES Science Gateway (Miller, Pfeiffer et al. 2011). The phylogeny was calculated using the PROTGAMMA protein substitution model, LG protein substitution matrix, and a parsimony seed value of 12345 . Ancestral sequences were calculated using CodeML, a maximum likelihood program from the PAML package, using the LG rate file with the Empirical+F model and using 8 categories in $\mathrm{dG}$ of NSsites models (Yang 1997). Ancestral Sequence Reconstructions at each node were used to determine G20/V20 identity at each node. Genomic context for the stators was was pulled from the KEGG Database (Kanehisa and Goto 2000).

\section{AUTHOR CONTRIBUTIONS}

PR and MABB designed experiments and executed experiments in strain editing, molecular biology and microbiology. TI and YS executed experiments in strain editing. MABB executed bioinformatics surrounding variant calling. $A L$ and $M A B B$ executed bioinformatics surrounding phylogenetics. PR, DTH, EG and MB executed bioinformatics surrounding the transcriptomics. MB supervised the design, execution and writing of the project. All authors contributed to writing and revision of the manuscript.

ACKNOWLEDGEMENTS

We would like to acknowledge Myu Yoshida and Rie Ito for technical assistance.

\section{FUNDING}

YS was supported by JSPS KAKENHI (JP18H02475 and JP20K06564), MEXT KAKENHI (JP19H05404 and JP 21H00410) and Takeda Science Foundation. MABB 
bioRxiv preprint doi: https://doi.org/10.1101/2021.01.26.427765; this version posted December $15,2021$. The copyright holder for this

preprint (which was not certified by peer review) is the author/funder, who has granted bioRxiv a license to display the preprint in perpetuity. It is made available under aCC-BY 4.0 International license.

433 Biology Future Science Platform 2018 Project Grant, and ARC Discovery Project

434 DP190100497.

435 
Anand, A., C. A. Olson, L. Yang, A. V. Sastry, E. Catoiu, K. S. Choudhary, P. V. Phaneuf, T. E. Sandberg, S. Xu, Y. Hefner, R. Szubin, A. M. Feist and B. O. Palsson (2019). "Pseudogene repair driven by selection pressure applied in experimental evolution." Nat Microbiol 4(3): 386-389. Andrews, S. (2010). FastQC: A Quality Control Tool for High Throughput Sequence Data [Online]. Available online at: http://www.bioinformatics.babraham.ac.uk/projects/fastqc/. Asai, Y., T. Shoji, I. Kawagishi and M. Homma (2000). "Cysteine-scanning mutagenesis of the periplasmic loop regions of PomA, a putative channel component of the sodium-driven flagellar motor in Vibrio alginolyticus." J Bacteriol 182(4): 1001-1007.

Asai, Y., T. Yakushi, I. Kawagishi and M. Homma (2003). "Ion-coupling determinants of Na+-driven and H+-driven flagellar motors." J Mol Biol 327(2): 453-463.

Atia, J., C. McCloskey, A. S. Shmygol, D. A. Rand, H. A. van den Berg and A. M. Blanks (2016). "Reconstruction of Cell Surface Densities of Ion Pumps, Exchangers, and Channels from mRNA Expression, Conductance Kinetics, Whole-Cell Calcium, and Current-Clamp Voltage Recordings, with an Application to Human Uterine Smooth Muscle Cells." PLoS Comput Biol 12(4): e1004828. Baym, M., T. D. Lieberman, E. D. Kelsic, R. Chait, R. Gross, I. Yelin and R. Kishony (2016). "Spatiotemporal microbial evolution on antibiotic landscapes." Science 353(6304): 1147-1151. Block, S. M., D. F. Blair and H. C. Berg (1989). "Compliance of bacterial flagella measured with optical tweezers." Nature 338(6215): 514-518.

Bloom, J. D. and F. H. Arnold (2009). "In the light of directed evolution: pathways of adaptive protein evolution." Proc Natl Acad Sci U S A 106 Suppl 1: 9995-10000.

Bush, S. J., D. Foster, D. W. Eyre, E. L. Clark, N. De Maio, L. P. Shaw, N. Stoesser, T. E. A. Peto, D. W. Crook and A. S. Walker (2020). "Genomic diversity affects the accuracy of bacterial single-nucleotide polymorphism-calling pipelines." Gigascience $\mathbf{9 ( 2 )}$.

460 Chen, S., Y. Zhou, Y. Chen and J. Gu (2018). "fastp: an ultra-fast all-in-one FASTQ preprocessor." 461 Bioinformatics 34(17): i884-i890. Colin, R. and V. Sourjik (2017). "Emergent properties of bacterial chemotaxis pathway." Curr Opin Microbiol 39: 24-33. Datsenko, K. A. and B. L. Wanner (2000). "One-step inactivation of chromosomal genes in Escherichia coli K-12 using PCR products." Proc Natl Acad Sci U S A 97(12): 6640-6645. Deme, J. C., S. Johnson, O. Vickery, A. Aron, H. Monkhouse, T. Griffiths, R. H. James, B. C. Berks, J. W. Coulton, P. J. Stansfeld and S. M. Lea (2020). "Structures of the stator complex that drives rotation of the bacterial flagellum." Nat Microbiol 5(12): 1553-1564.

Deme, J. C., S. Johnson, O. Vickery, A. Muellbauer, H. Monkhouse, T. Griffiths, R. H. James, B. C. Berks, J. W. Coulton, P. J. Stansfeld and S. M. Lea (2020). "Structures of the stator complex that drives rotation of the bacterial flagellum." Nature Microbiology 5(12): 1553-+.

Draghi, J. A. and J. B. Plotkin (2011). "Molecular evolution: Hidden diversity sparks adaptation." Nature 474(7349): 45-46.

Foster, P. L., H. Lee, E. Popodi, J. P. Townes and H. Tang (2015). "Determinants of spontaneous mutation in the bacterium Escherichia coli as revealed by whole-genome sequencing." Proc Natl Acad Sci U S A 112(44): E5990-5999.

Fraebel, D. T., H. Mickalide, D. Schnitkey, J. Merritt, T. E. Kuhlman and S. Kuehn (2017).

"Environment determines evolutionary trajectory in a constrained phenotypic space." Elife 6.

Garza, A. G., L. W. Harris-Haller, R. A. Stoebner and M. D. Manson (1995). "Motility protein interactions in the bacterial flagellar motor." Proc Natl Acad Sci U S A 92(6): 1970-1974.

Gude, S., E. Pince, K. M. Taute, A. B. Seinen, T. S. Shimizu and S. J. Tans (2020). "Bacterial coexistence driven by motility and spatial competition." Nature 578(7796): 588-592.

Hase, C. C., N. D. Fedorova, M. Y. Galperin and P. A. Dibrov (2001). "Sodium ion cycle in bacterial pathogens: evidence from cross-genome comparisons." Microbiol Mol Biol Rev 65(3): 353-370, table of contents.

Hayden, E. J., E. Ferrada and A. Wagner (2011). "Cryptic genetic variation promotes rapid evolutionary adaptation in an RNA enzyme." Nature 474(7349): 92-95. 
Higuchi, R., B. Krummel and R. K. Saiki (1988). "A general method of in vitro preparation and specific mutagenesis of DNA fragments: study of protein and DNA interactions." Nucleic Acids Res 16(15): 7351-7367. Ishida, T., R. Ito, J. Clark, N. J. Matzke, Y. Sowa and M. A. B. Baker (2019). "Sodium-powered stators of the bacterial flagellar motor can generate torque in the presence of phenamil with mutations near the peptidoglycan-binding region." Mol Microbiol 111(6): 1689-1699. Islam, M. I., A. Lin, Y. W. Lai, N. J. Matzke and M. A. B. Baker (2020). "Ancestral Sequence Reconstructions of MotB Are Proton-Motile and Require MotA for Motility." Front Microbiol 11: 625837. Jaques, S., Y. K. Kim and L. L. McCarter (1999). "Mutations conferring resistance to phenamil and amiloride, inhibitors of sodium-driven motility of Vibrio parahaemolyticus." Proc Natl Acad Sci U SA 96(10): 5740-5745. Kanehisa, M. and S. Goto (2000). "KEGG: kyoto encyclopedia of genes and genomes." Nucleic Acids Res 28(1): 27-30.

Karp, P. D., M. Riley, M. Saier, I. T. Paulsen, J. Collado-Vides, S. M. Paley, A. Pellegrini-Toole, C. Bonavides and S. Gama-Castro (2002). "The EcoCyc Database." Nucleic Acids Res 30(1): 56-58. Kim, D., B. Langmead and S. L. Salzberg (2015). "HISAT: a fast spliced aligner with low memory requirements." Nat Methods 12(4): 357-360.

Kinosita, Y., T. Ishida, M. Yoshida, R. Ito, Y. V. Morimoto, K. Goto, R. M. Berry, T. Nishizaka and Y. Sowa (2020). "Distinct chemotactic behavior in the original Escherichia coli K-12 depending on forward-and-backward swimming, not on run-tumble movements." Sci Rep 10(1): 15887. Kojima, S., Y. Asai, T. Atsumi, I. Kawagishi and M. Homma (1999). "Na+-driven flagellar motor resistant to phenamil, an amiloride analog, caused by mutations in putative channel components." J Mol Biol 285(4): 1537-1547.

Kojima, S., M. Kuroda, I. Kawagishi and M. Homma (1999). "Random mutagenesis of the pomA gene encoding a putative channel component of the $\mathrm{Na}(+)$-driven polar flagellar motor of Vibrio alginolyticus." Microbiology (Reading) 145 ( Pt 7): 1759-1767.

Kojima, S., T. Shoji, Y. Asai, I. Kawagishi and M. Homma (2000). "A slow-motility phenotype caused by substitutions at residue Asp31 in the PomA channel component of a sodium-driven flagellar motor." J Bacteriol 182(11): 3314-3318.

518 Lai, Y. W., P. Ridone, G. Peralta, M. M. Tanaka and M. A. B. Baker (2020). "Evolution of the Stator 519 Elements of Rotary Prokaryote Motors." J Bacteriol 202(3).

520 Lopatkin, A. J. and J. H. Yang (2021). "Digital Insights Into Nucleotide Metabolism and Antibiotic 521 Treatment Failure." Front Digit Health 3. RNA-seq data with DESeq2." Genome Biol 15(12): 550.

Mandadapu, K. K., J. A. Nirody, R. M. Berry and G. Oster (2015). "Mechanics of torque generation in the bacterial flagellar motor." Proc Natl Acad Sci U S A 112(32): E4381-4389.

McDonald, M. J. (2019). "Microbial Experimental Evolution - a proving ground for evolutionary theory and a tool for discovery." EMBO Rep 20(8): e46992.

Miller, M. A., W. Pfeiffer and T. Schwartz (2011). The CIPRES science gateway: a community resource for phylogenetic analyses. Proceedings of the 2011 TeraGrid Conference: extreme digital discovery. Mirdita, M., K. Schütze, Y. Moriwaki, L. Heo, S. Ovchinnikov and M. Steinegger (2021). "ColabFold Making protein folding accessible to all." 2021.2008.2015.456425.

Morgan M, P. H., Obenchain V, Hayden N. (2021). " Rsamtools: Binary alignment (BAM), FASTA, variant call (BCF), and tabix file import. R package version 2.10.0."

https://bioconductor.org/packages/Rsamtools. .

Mulkidjanian, A. Y., M. Y. Galperin, K. S. Makarova, Y. I. Wolf and E. V. Koonin (2008). "Evolutionary primacy of sodium bioenergetics." Biol Direct 3: 13.

Ni, B., R. Colin, H. Link, R. G. Endres and V. Sourjik (2020). "Growth-rate dependent resource investment in bacterial motile behavior quantitatively follows potential benefit of chemotaxis." Proc Natl Acad Sci U S A 117(1): 595-601.

Ni, B., B. Ghosh, F. S. Paldy, R. Colin, T. Heimerl and V. Sourjik (2017). "Evolutionary Remodeling of Bacterial Motility Checkpoint Control." Cell Rep 18(4): 866-877. 
Nishino, Y., Y. Onoue, S. Kojima and M. Homma (2015). "Functional chimeras of flagellar stator proteins between E-coli MotB and Vibrio PomB at the periplasmic region in Vibrio or E-coli." Microbiologyopen 4(2): 323-331.

Nishiyama, M. and S. Kojima (2012). "Bacterial motility measured by a miniature chamber for highpressure microscopy." Int J Mol Sci 13(7): 9225-9239. Pallen, M. J. and N. J. Matzke (2006). "From The Origin of Species to the origin of bacterial flagella." $548 \quad$ Nat Rev Microbiol 4(10): 784-790.

549 Pang, B., J. L. McFaline, N. E. Burgis, M. Dong, K. Taghizadeh, M. R. Sullivan, C. E. Elmquist, R. P. 550 Cunningham and P. C. Dedon (2012). "Defects in purine nucleotide metabolism lead to substantial 551 incorporation of xanthine and hypoxanthine into DNA and RNA." Proc Natl Acad Sci U S A 109(7): 2319-2324.

Parkinson, J. S. (1978). "Complementation analysis and deletion mapping of Escherichia coli mutants defective in chemotaxis." J Bacteriol 135(1): 45-53.

Pertea, M., G. M. Pertea, C. M. Antonescu, T. C. Chang, J. T. Mendell and S. L. Salzberg (2015).

"StringTie enables improved reconstruction of a transcriptome from RNA-seq reads." Nat Biotechnol 33(3): 290-295.

Reisch, C. R. and K. L. Prather (2015). "The no-SCAR (Scarless Cas9 Assisted Recombineering) system for genome editing in Escherichia coli." Sci Rep 5: 15096.

Roszak, D. B. and R. R. Colwell (1987). "Survival strategies of bacteria in the natural environment." Microbiol Rev 51(3): 365-379.

Santiveri, M., A. Roa-Eguiara, C. Kühne, N. Wadhwa, H. C. Berg, M. Erhardt and N. M. I. Taylor (2020). "Structure and function of stator units of the bacterial flagellar motor." 2020.2005.2015.096610. Stamatakis, A. (2014). "RAxML version 8: a tool for phylogenetic analysis and post-analysis of large phylogenies." Bioinformatics (Oxford, England) 30(9): 1312-1313.

Sudo, Y., H. Terashima, R. Abe-Yoshizumi, S. Kojima and M. Homma (2009). "Comparative study of the ion flux pathway in stator units of proton- and sodium-driven flagellar motors." Biophysics (Nagoya-shi) 5: 45-52.

Takekawa, N., M. Isumi, H. Terashima, S. Zhu, Y. Nishino, M. Sakuma, S. Kojima, M. Homma and K. Imada (2019). "Structure of Vibrio FliL, a New Stomatin-like Protein That Assists the Bacterial Flagellar Motor Function." mBio 10(2).

Takekawa, N., M. Nishiyama, T. Kaneseki, T. Kanai, H. Atomi, S. Kojima and M. Homma (2015). "Sodium-driven energy conversion for flagellar rotation of the earliest divergent hyperthermophilic bacterium." Sci Rep 5: 12711.

Taylor, T. B., G. Mulley, A. H. Dills, A. S. Alsohim, L. J. McGuffin, D. J. Studholme, M. W. Silby, M. A. Brockhurst, L. J. Johnson and R. W. Jackson (2015). "Evolution. Evolutionary resurrection of flagellar motility via rewiring of the nitrogen regulation system." Science 347(6225): 1014-1017.

Terahara, N., T. A. Krulwich and M. Ito (2008). "Mutations alter the sodium versus proton use of a Bacillus clausii flagellar motor and confer dual ion use on Bacillus subtilis motors." Proceedings of the National Academy of Sciences of the United States of America 105(38): 14359-14364.

Terahara, N., M. Sano and M. Ito (2012). "A Bacillus Flagellar Motor That Can Use Both Na+ and K+ as a Coupling Ion Is Converted by a Single Mutation to Use Only Na+." Plos One 7(9).

Yang, Z. (1997). "PAML: a program package for phylogenetic analysis by maximum likelihood." Computer applications in the biosciences 13(5): 555-556. 
A

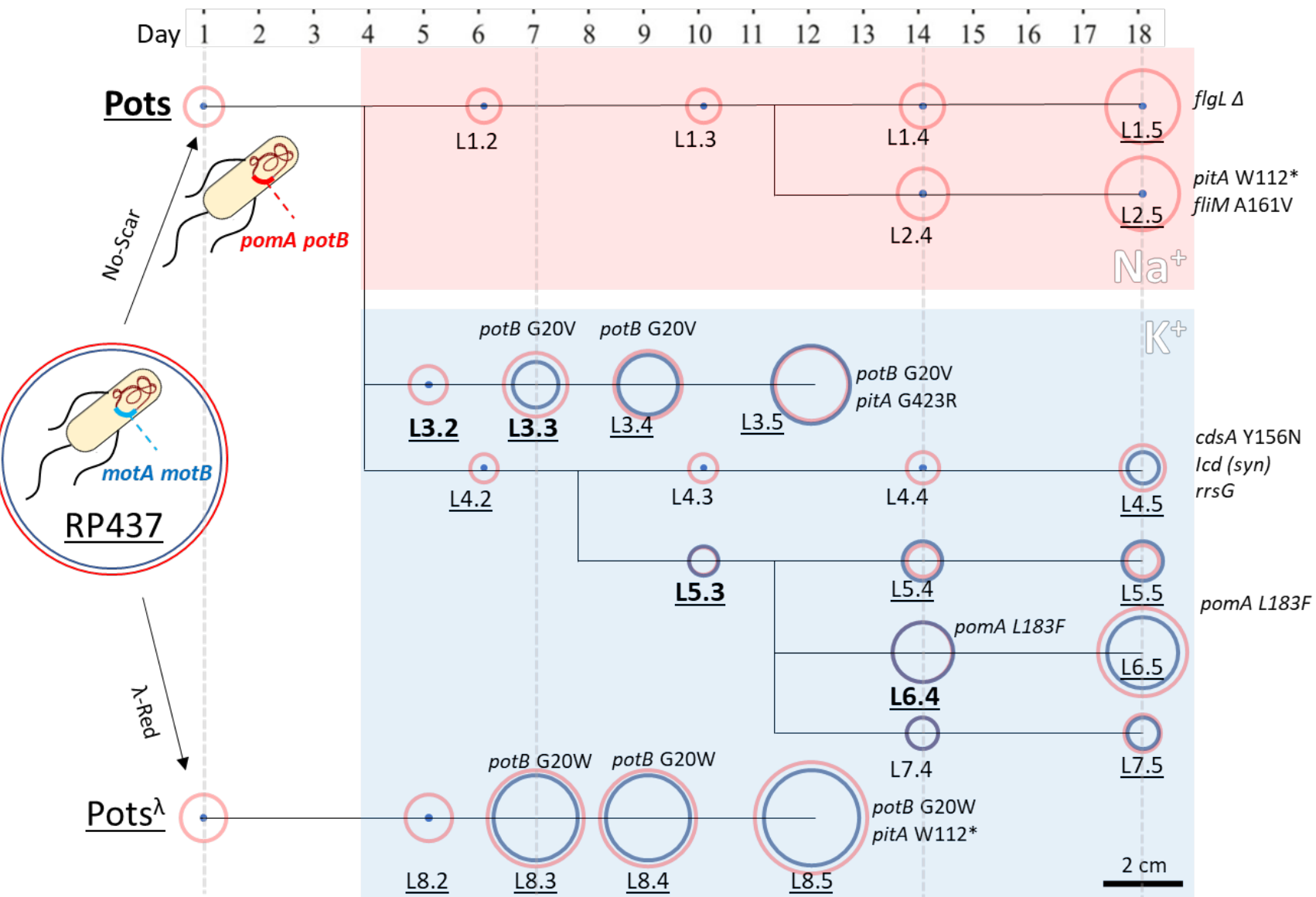

B
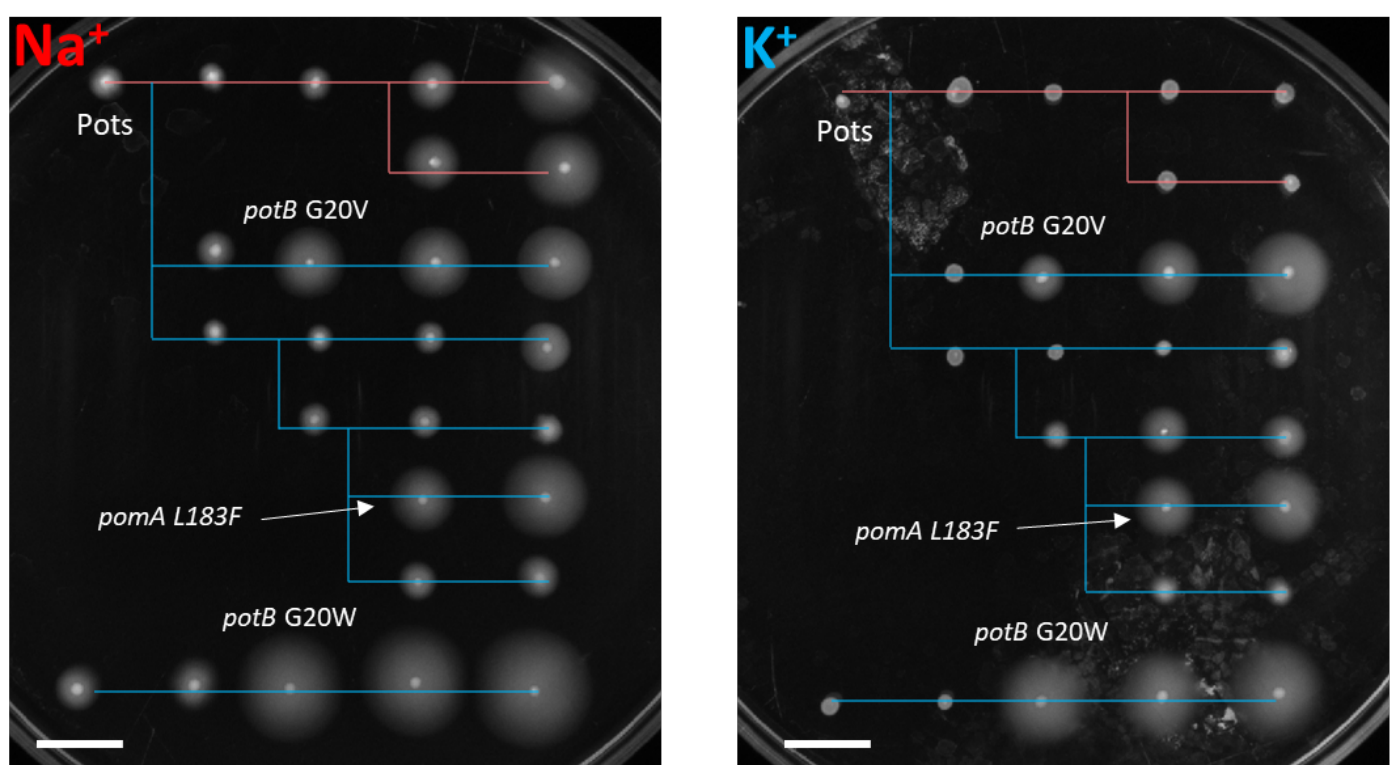

Figure 1. Directed Evolution of the Flagellar Motor

A) Summary of the directed evolution experiments. The edited E. coli strains Pots and Pots ${ }^{\lambda}$, obtained from E. coli RP437 via No-Scar and $\lambda$-Red recombineering respectively, were passaged on soft agar (colored background, red: $\mathrm{Na}+$; blue: $\mathrm{K}+$ ) over an 18-day period. The summary comprises a total of 8 lineages (L1-L8) selected for further investigation, each comprised of 5 members (ie. L1.3 indicates the first lineage and the third passage of a motile flare). The day of collection and reinoculation is indicated above each lineage member. The ability of each lineage 
member to swim in the presence of either $\mathrm{Na}+$ or $\mathrm{K}+$ is displayed by a red or blue ring, respectively, corresponding to swim size on a swim plate. Lack of motility after $24 \mathrm{hr}$ of incubation on $\mathrm{K}+$ soft agar is represented by a blue dot. Ring sizes were measured from pair of large swim plates presented in panel B. Member labels are underscored to indicate WGS data availability and bolded to indicate RNAseq data availability. All strains were locally Sanger sequenced at the pomApotB locus, but 21 strains (underscored) underwent whole genome sequencing (WGS ). The full list of SNPs identified via variant calling relative to the Pots reference genome are annotated next to each respective lineage member. Highlighted genes other than pomA and potB: pitA (metal phosphate: $\mathrm{H}^{+}$symporter), flgL (flagellar hook-filament junction protein 2), fliM (flagellar motor switch protein), cdsA (cardiolipin-diglyceride synthase), icd (isocitrate dehydrogenase), rrsG (16S ribosomal RNA). Black scale bar $=2 \mathrm{~cm}$.

B) Recapitulation of the directed evolution experiment. $\mathrm{Na}+$ (left) and $\mathrm{K}+$ (right) soft agar plates inoculated with a $1 \mu \mathrm{l}$ aliquot of glycerol stock of each strain indicated in A (except RP437 [Supplementary Fig. 5]) and arranged in the same order as $(A)$ on a large petri dish $(\varnothing=30 \mathrm{~cm})$. The colored lines indicate the type of soft agar each lineage was propagated in and isolated from. Arrows and labels indicate the first member in each lineage to display a mutation in the stator genes PomA or PotB. Each plate was incubated for $24 \mathrm{hr}$ at $30^{\circ} \mathrm{C}$. White scale bar $=2 \mathrm{~cm}$. 
A

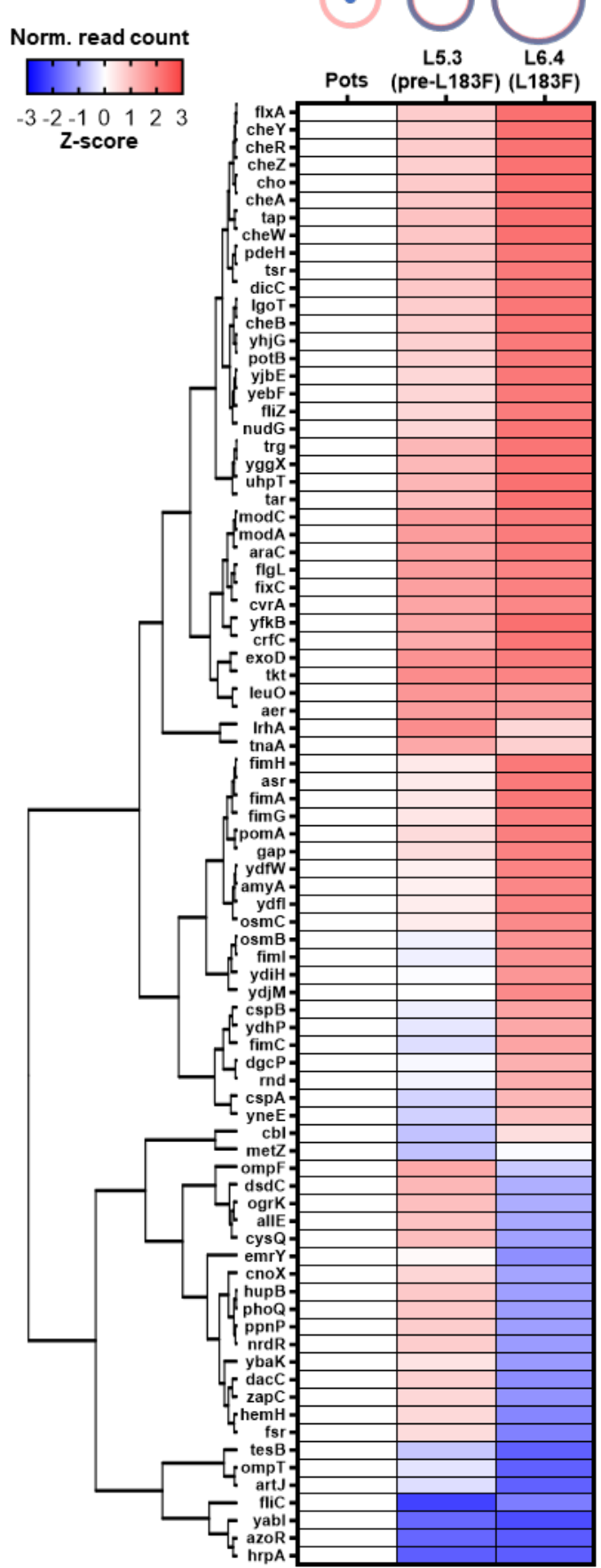

C

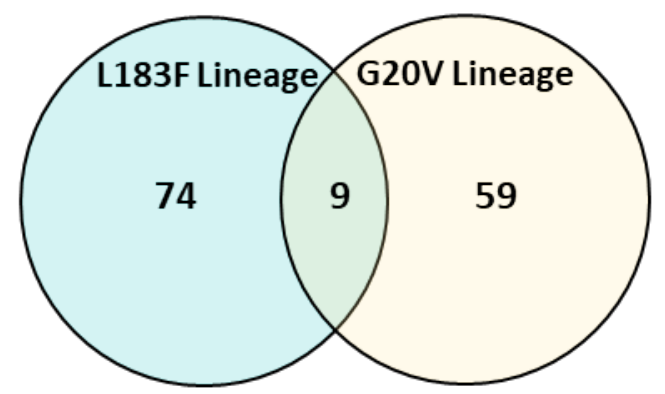

B
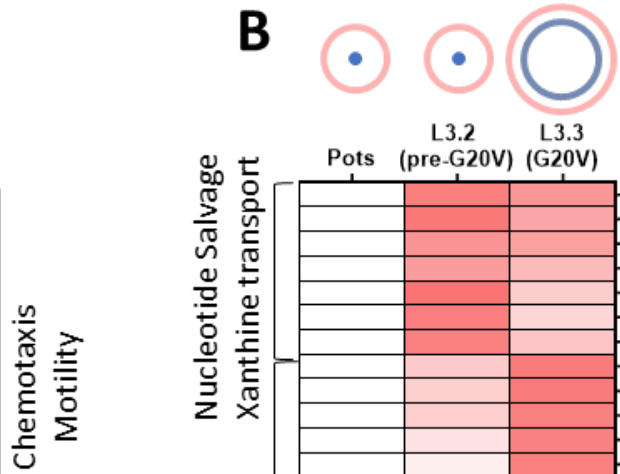

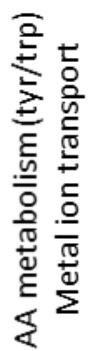

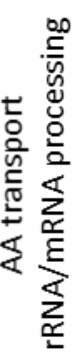

$\therefore$ Pots (pre-G20V) (G20V)
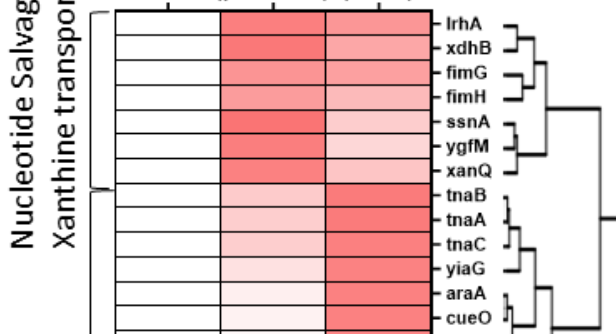

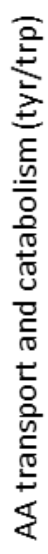

足
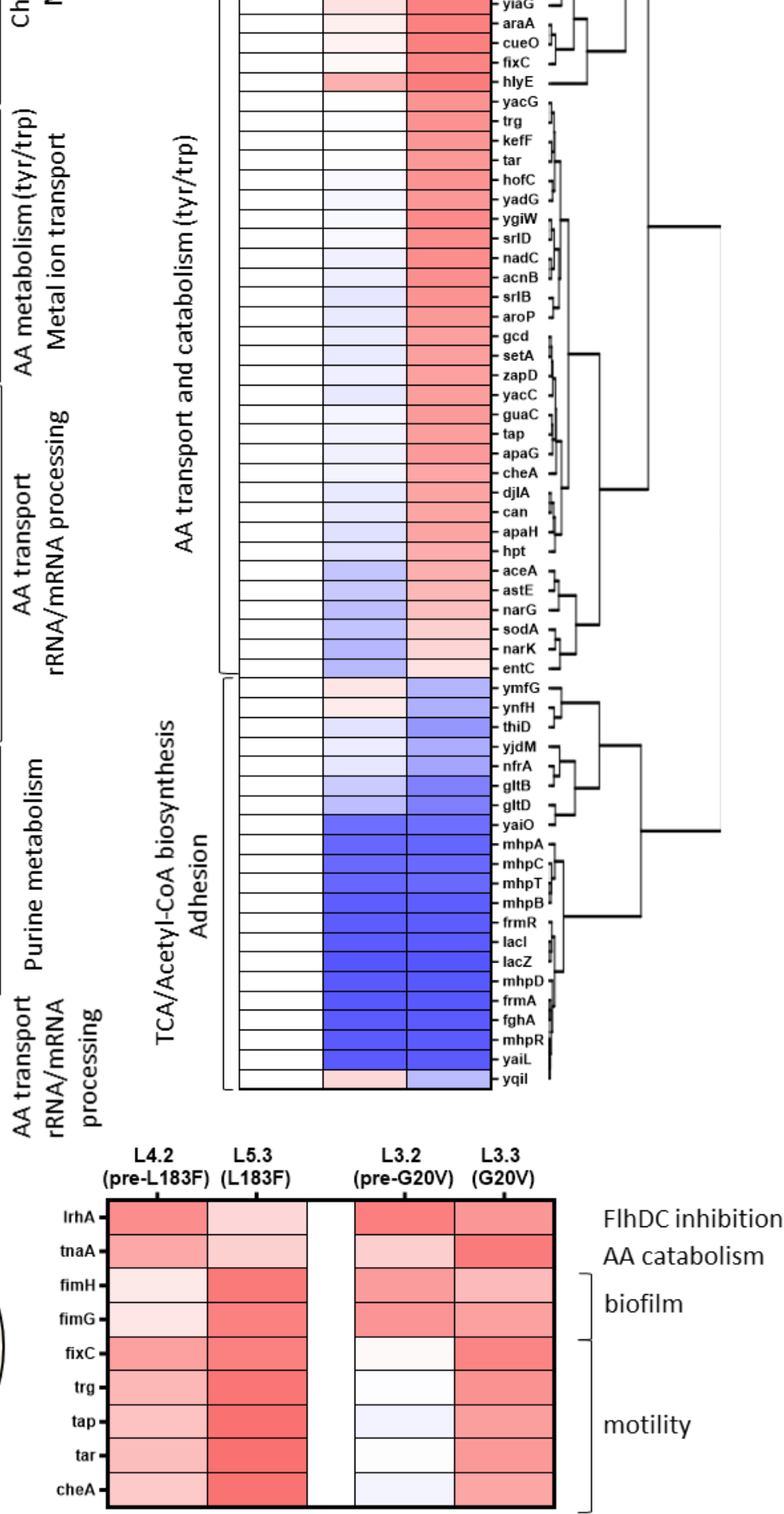

Fig.2. Differential expression analysis of RNAseq data. Differentially expressed 
619 L183F lineage (A) or G20V lineage (B). The average read counts $(n=3)$ for each 620 gene were normalized by z-score and displayed as clustered heatmaps, flanked by 621 their respective dendrograms. DEG clusters are labelled by their representative 622 biological process (PANTHER). Genes upregulated with respect to the Pots strain 623 are labelled in red, downregulated ones in blue. The ring diagrams above each 624 heatmap are taken from fig. 1 and indicate the motility phenotype of each lineage 625 member. C) Venn diagram indicating the number of DEGs in each dataset and the 626 DEGs in common, shown in the heatmap on the right. 

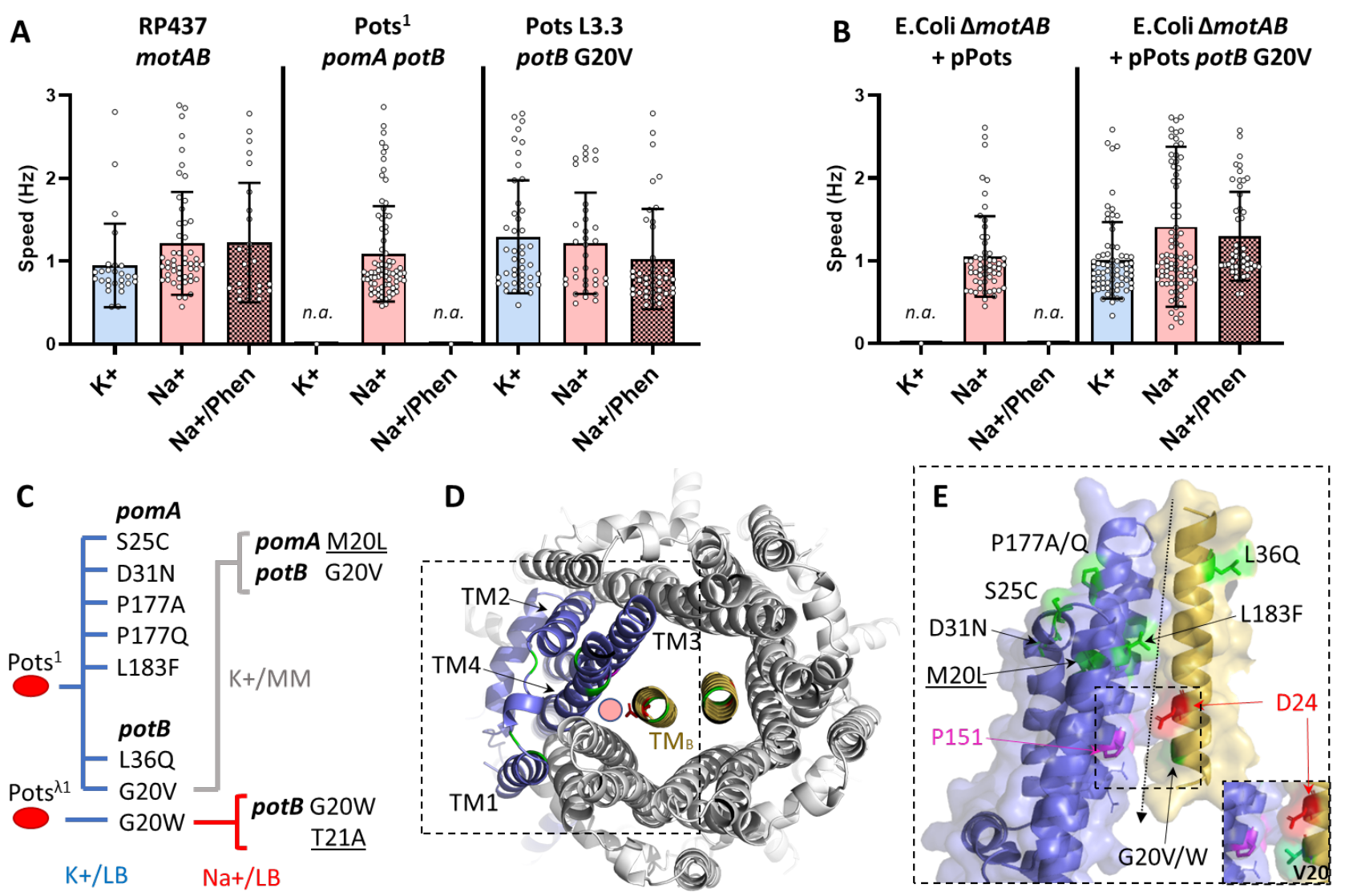

630 Figure 3. Functional characterization of evolved stators. A) Single cell speed measurements using the tethered cell assay measured in $\mathrm{Hz}$ (revolutions/s). Blue bar indicates speed in $67 \mathrm{mM} \mathrm{KCl}$ motility Buffer, red bar: $85 \mathrm{mM} \mathrm{NaCl}$ motility Buffer; red patterned bar: $85 \mathrm{mM} \mathrm{NaCL}+100 \mu \mathrm{M}$ phenamil motility buffer. Number of cells analysed per condition (from left to right): RP437: 27, 51, 25; pots: n.a, 78, n.a; pots potB G20V 45, 36, 39 (n.a. indicates no visible rotating cell). Error bars indicate Standard Deviation (S.D.). B) Single cell speed measurements using the tethered cell assay in RP6894 $\triangle$ motAB strain co-expressing pomA and potB G20V from pPots plasmid. Blue bar indicates speed in $67 \mathrm{mM} \mathrm{KCl}$ motility buffer, red bar: $85 \mathrm{mM} \mathrm{NaCl}$ motility buffer; red patterned bar: $85 \mathrm{mM} \mathrm{NaCl}+100 \mu \mathrm{M}$ phenamil motility buffer. Number of cells analysed per condition (from left to right): ( $\triangle \operatorname{mot} A B+$ pPots: n.a., 32 , n.a; $\triangle \operatorname{mot} A B+$ pPots potB G20V: 40, 63, 48). Error bars indicate S.D. Single cell tracked data shown in Supplementary Fig. 12. C) Graphical summary of stator gene mutations detected across all directed evolution experiments and the growth conditions under which these mutations arose. LB indicates agar containing yeast extract and tryptone. MM indicates agar in minimal media. Mutations in a subsequent generation are underlined. D) View from the extracellular side of the transmembrane portion of PomA $\mathrm{A}_{5} \mathrm{PotB}_{2}$ stator complex (see Methods: Structural Modelling). One monomer of subunit $A$ is coloured in blue and the TM domains of the $B$ subunits are 
649 coloured in yellow. Mutant sites obtained in the directed evolution experiments are 650 labelled in green, the catalytic aspartate residue essential for function is highlighted in 651 red. The red circle indicates the predicted location of the ion transport pore (inward 652 conduction). E) side view of the area highlighted by the dashed box in $\mathrm{D}$. Residues $653 \mathrm{P} 151$ (PomA) and D24 (PotB) are also highlighted in magenta and red respectively. 654 Primary mutation sites are indicated in black, while secondary mutation sites are 655 underscored. The arrow at the interface between (A)TM3-4 and (B)TM indicates the 656 predicted location of the ion transport pore. The inset highlights the change in the pore 657 region due to the G20V substitution in PotB (green). 